\title{
Von Willebrand Factor and Von Willebrand Factor-Cleaving Metalloprotease Activity in Escherichia coli O157:H7-Associated Hemolytic Uremic Syndrome
}

\author{
HAN-MOU TSAI, WAYNE L. CHANDLER, RAVINDRA SARODE, ROBERT HOFFMAN, \\ SRDJAN JELACIC, REBECCA L. HABEEB, SANDRA L. WATKINS, CRAIG S. WONG, \\ GLYN D. WILLIAMS, AND PHILLIP I. TARR
}

\begin{abstract}
Montefiore Medical Center/Albert Einstein College of Medicine, Division of Hematology, Bronx, NY 10467, U.S.A. [H.-M.T.]; University of Washington School of Medicine, Laboratory Medicine, Hematology and Coagulation, Seattle, WA 98015, U.S.A. [W.L.C.]; University Hospitals of Cleveland/Case Western Reserve University School of Medicine, Department of Pathology, Cleveland, OH 44106, U.S.A. [R.S., R.H.]; University of Washington and Children's Hospital and Regional Medical Center, Divisions of Gastroenterology [S.J., R.L.H., P.I.T.], Nephrology [S.L.W., C.S.W.], and Anesthesiology [G.D.W.], Seattle, WA 98105, U.S.A.
\end{abstract}

\section{ABSTRACT}

\begin{abstract}
Hemolytic uremic syndrome (HUS) usually occurs after infection with Shiga toxin-producing bacteria. Thrombotic thrombocytopenic purpura, a disorder with similar clinical manifestations, is associated with deficient activity of a circulating metalloprotease that cleaves von Willebrand factor at the Tyr842-Met843 peptide bond in a shear stress-dependent manner. We analyzed von Willebrand factor-cleaving metalloprotease activity and the status of von Willebrand factor in 16 children who developed HUS after Escherichia coli O157:H7 infection and in 29 infected children who did not develop this complication. Von Willebrand factor-cleaving metalloprotease activity was normal in all subjects, but von Willebrand factor size was decreased in the plasma of each of 16 patients with HUS. The decrease in circulating von Willebrand factor size correlated with the severity of thrombocytopenia and was proportional to an increase in von Willebrand factor proteolytic fragments in
\end{abstract}

plasma. Immunohistochemical studies of the kidneys in four additional patients who died of HUS demonstrated glomerular thrombi in three patients, and arterial and arteriolar thrombi in one patient. The glomerular thrombi contained fibrin but little or no von Willebrand factor. A decrease in large von Willebrand factor multimers, presumably caused by enhanced proteolysis from abnormal shear stress in the microcirculation, is common in HUS. (Pediatr Res 49: 653-659, 2001)

$\quad$ Abbreviations:
HUS, hemolytic uremic syndrome
Met, methionine
stx, Shiga toxin gene
TTP, thrombotic thrombocytopenic purpura
Tyr, tyrosine

HUS, characterized by acute renal failure, hemolysis with schistocytes on blood smears, and thrombocytopenia, is accompanied by thrombotic microangiopathy of the kidneys and of other organs (1). The syndrome covers a diverse spectrum of microangiopathic disorders (2-4), but most cases occur after infection with Shiga toxin-producing bacteria, such as Escherichia coli O157:H7 (5) or Shigella dysenteriae serotype 1 (6).

TTP, a disorder with some clinical, laboratory, and histopathologic similarities to HUS, has been associated with

Received June 16, 2000; accepted November 29, 2000

Correspondence and reprint requests: Han-Mou Tsai, M.D., Montefiore Medical Center, Division of Hematology, 111 East 210th Street, Bronx, NY 10467, U.S.A.; e-mail: htsai@montefiore.org

Supported by NIH grants R01 HL 62131 (H.-M.T.) and R01 DK 52081 (P.I.T.). abnormal homeostasis of von Willebrand factor, a protein that is secreted from endothelial cells as a disulfide-linked polymer of a polypeptide with 2050 amino acid residues. Circulating von Willebrand factor is normally cleaved between Tyr842 and Met843 (7) in a shear stress-dependent manner $(8)$ by a plasma metalloprotease $(9,10)$, generating a series of multimers. Without this metalloprotease activity, von Willebrand factor, when unfolded by shear stress (11), has increased platelet-aggregating activity (12). It is postulated that this increased activity facilitates the formation of arteriolar and capillary platelet thrombi in TTP. Indeed, acquired TTP has been associated with deficient von Willebrand factor-cleaving metalloprotease activity caused by inhibitory antibodies $(12,13)$. 
Aberrations in von Willebrand factor multimers have been described in HUS (14-17), but these reports did not adequately characterize the cause of the thrombotic microangiopathy in the subjects studied. One study reports that HUS is not associated with deficient von Willebrand factor-cleaving metalloprotease activity (13). However, this study also did not indicate the cause of the HUS in the subjects. To explore further the role of von Willebrand factor in the pathogenesis of HUS, we investigated von Willebrand factor and its cleaving metalloprotease activity in children with documented E. coli $\mathrm{O} 157: \mathrm{H} 7$ infection, with and without microangiopathic sequelae.

\section{METHODS}

Subjects. Children infected with E. coli O157:H7 were enrolled via a laboratory-based identification system in Washington, Oregon, Idaho, and Wyoming (18).

Infected children were considered to have HUS if they had hemolytic anemia (hematocrit $<0.30$ with evidence of microangiopathic changes on peripheral smears), thrombocytopenia (platelet count $<150 \times 10^{9} / \mathrm{L}$ ), and renal insufficiency (creatinine $>$ upper limit of normal for age). Infected children were considered to have uncomplicated illness if they did not develop HUS. Children with confirmed E. coli O157:H7 infection and HUS who had not been enrolled in the study during the prodromal stage were also enrolled as subjects. Control plasma samples were obtained from children aged $<10 \mathrm{y}$ without inflammatory, hematologic, infectious, or nephrologic disorders, and who were undergoing elective surgery, usually to repair inguinal hernias.

Parental informed consent was obtained for each subject. Informed assent was obtained from patients and controls, if appropriate for age and mental status. Institutional review board approval was obtained at each participating hospital.

The first day of diarrhea was considered to be the first day of illness. The duration of thrombocytopenia is considered to be the interval between the first day of HUS, and the first of two consecutive days in which the platelet count rose without platelet transfusions.

Toxin genotyping of E. coli O157:H7 strains. DNA from each isolate was digested (BamHI), electrophoretically separated, transferred to a nylon membrane (Micron Separations, Westboro, MA, U.S.A.), and probed under stringent conditions (19) with the inserts of pJN37-19 and pNN111-19 to identify stx 1 and stx2, respectively (20). DNA samples from E. coli O103:H2 (21) and E. coli O157:H7 strain 86-24 (22) were included as positive controls for stx 1 and stx2, respectively. $E$. coli HB101 (19) served as the negative control.

Plasma. Blood from subjects obtained by phlebotomy (1.8 $\mathrm{mL}$ ) was anticoagulated by adding $0.2 \mathrm{~mL}$ of sodium citrate $(0.105 \mathrm{M})$, placed on ice, and centrifuged within $1 \mathrm{~h}$. Plasma was divided into aliquots, and frozen at $-70^{\circ} \mathrm{C}$.

Analysis of von Willebrand factor-cleaving metalloprotease activity and von Willebrand factor proteolysis. Von Willebrand factor antigen concentration and von Willebrand factor multimers were determined by enzyme immunoassay and SDS agarose gel electrophoresis (12). The fraction of large multimers, defined as those bands greater in size than the four smallest bands, was determined by scanning densitometry and expressed as a percentage of the large multimer fraction in pooled normal control plasma on the same gel.

Dimers of the $176-\mathrm{kD}$ and the $140-\mathrm{kD}$ fragments in plasma samples were separated by SDS-PAGE under nonreducing conditions, and visualized by immunoblotting using a polyclonal anti-von Willebrand factor antibody and an ${ }^{125}$ I-labeled donkey anti-rabbit IgG antibody, followed by autoradiography (12). These dimers appeared as $350-\mathrm{kD}$ and $200-\mathrm{kD}$ bands on the nonreducing gels, and were quantified by scanning densitometry and expressed as a percentage of the OD of the corresponding species in pooled normal control plasma.

The assay of von Willebrand factor-cleaving metalloprotease activity was based on the generation, from exogenous von Willebrand factor substrate, of dimers of the $176-\mathrm{kD}$ and the $140-\mathrm{kD}$ fragments (12). Normal control plasma obtained from the clinical coagulation laboratory was used as a reference and assumed to contain $100 \%$ of the metalloprotease activity.

Histochemical and immunochemical studies. Postmortem renal sections from four children who died of HUS were examined for thrombi by hematoxylin and eosin staining. $E$. coli $\mathrm{O} 157: \mathrm{H} 7$ was isolated from the stools of three of these children, and a Shiga toxin-producing E. coli was isolated from the stool of the fourth child whose HUS was preceded by hemorrhagic colitis, but serotype information on this isolate was not available. The lesions were examined for platelets, fibrin, or both, following the procedure of Carstairs (23). Fibrin, platelets, and erythrocytes stain pink, blue-gray, and yellow, respectively. Von Willebrand factor was detected by probing with a rabbit polyclonal anti-von Willebrand factor antibody. Bound antibody was detected with a biotinylated goat anti-rabbit IgG, peroxidase-conjugated streptavidin, and 3,3'-diaminobenzidine according to the manufacturer's instructions (Dako Corp, Carpinteria, CA, U.S.A.).

Statistics. Data in a group were expressed as mean \pm SD. Differences between groups were analyzed by ANOVA, or paired $t$ test when appropriate. The correlation coefficient between two variables was determined by using the Pearson product-moment method (24).

\section{RESULTS}

Clinical features. Thirty-seven patients were enrolled and investigated at the colitis stage of E. coli $\mathrm{O} 157: \mathrm{H} 7$ infection. Eight of these patients subsequently developed HUS. Eight additional cases were studied only at the HUS stage, because plasma samples at the colitis stage were not available. Ten of the 16 HUS patients required dialysis; none of the patients received plasma infusion or exchange. All patients survived. Case features are summarized in Table 1.

Von Willebrand factor-cleaving metalloprotease activity. The metalloprotease activities in one HUS, one TTP, and one normal control plasma sample are demonstrated in Figure $1 \mathrm{~A}$. Each sample was tested with and without EDTA, as indicated. In normal and in HUS plasma, samples without EDTA, compared with those with EDTA, generated $350-\mathrm{kD}$ and $200-\mathrm{kD}$ proteolytic fragments from the von Willebrand factor substrate. 
Table 1. Clinical and laboratory features

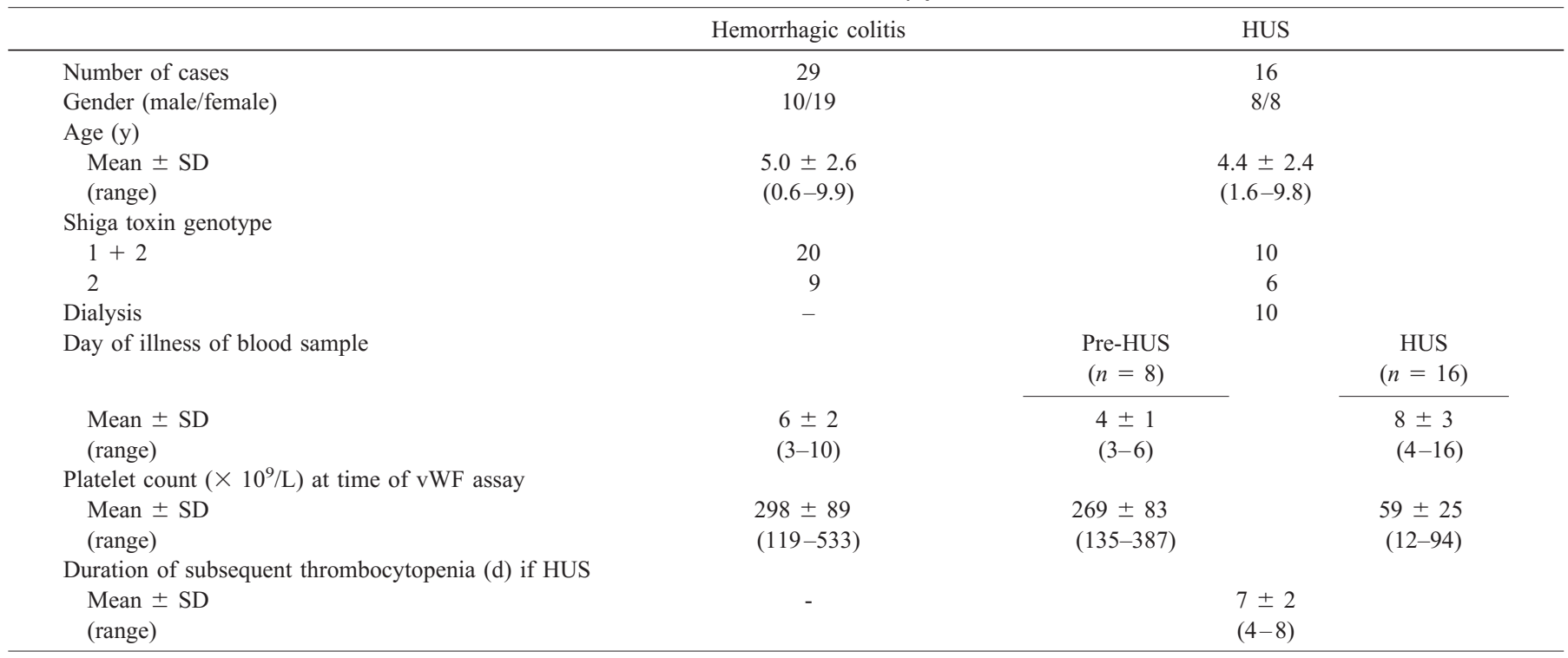

vWF, von Willebrand factor.

In contrast, few $(<10 \%)$ proteolytic fragments were generated in the TTP plasma, demonstrating the severely deficient plasma metalloprotease activity in TTP.

In the 16 HUS plasma samples, metalloprotease activity (mean $\pm \mathrm{SD}$ ) was $97 \pm 11 \%$ (range, 83 to $122 \%$ ), whereas it was $97 \pm 14 \%$ (range, 75 to $137 \%$ ) in the 37 plasma samples obtained at the colitis stage. The distribution of the metalloprotease activities in these patients and in seven normal children $(113 \pm 21 \%$; range, 92 to $152 \%)$ is shown in Figure $1 B$. In the 29 cases of uncomplicated hemorrhagic colitis, the metalloprotease activity was $96 \pm 15 \%$; of the eight patients who subsequently developed HUS, the metalloprotease activity was $98 \pm 9 \%$. These values are not significantly different from

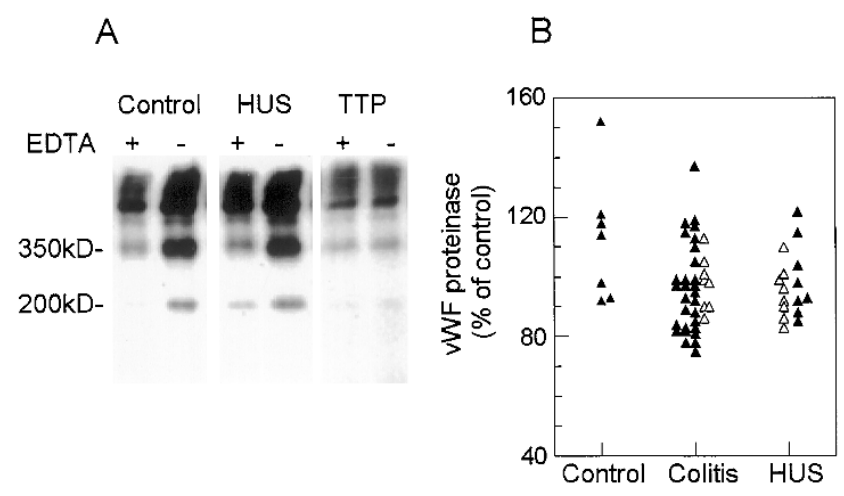

Fig. 1. Normal levels of von Willebrand factor cleaving metalloprotease activity in $E$. coli O157: $\mathrm{H} 7$ infection. $A$, immunoblot of SDS-PAGE depicting the generation of $350-\mathrm{kD}$ and $200-\mathrm{kD}$ fragments from von Willebrand factor substrate in the absence of EDTA in control (metalloprotease activity $=100 \%$ ) and HUS (metalloprotease activity $=88 \%$ ) samples. There is minimal generation of these fragments by a TTP plasma sample (metalloprotease activity < $10 \%)$. The $350-\mathrm{kD}$ and $200-\mathrm{kD}$ fragments in the EDTA lanes represented endogenous fragments in the plasma samples and the von Willebrand factor substrate. $B$, levels of von Willebrand factor $(v W F)$ cleaving metalloprotease activity in seven normal, 37 colitis, and 16 HUS plasma samples. The data of the eight patients who were studied at both colitis and HUS stages are represented by $\triangle$. each other and are within the range observed in 57 normal adult individuals without TTP $(103 \pm 12 \%)$ in a separate study $(25)$. Thus, no deficiency in von Willebrand factor-cleaving metalloprotease activity was detected in children infected with $E$. coli O157:H7, with or without HUS, unlike the deficiency observed in TTP.

Von Willebrand factor multimers. The von Willebrand factor multimers analyzed by SDS agarose gel electrophoresis in one patient who developed HUS are illustrated in Figure $2 A$.
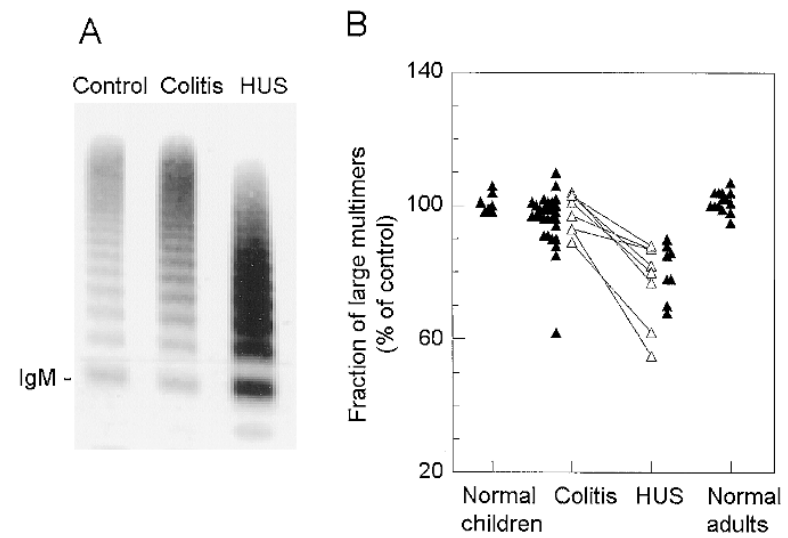

Fig. 2. Decrease of large von Willebrand factor multimers in HUS caused by $E$. coli $\mathrm{O} 157$ : $\mathrm{H} 7$ infection. $A$, autoradiograph of an SDS agarose gel depicting a loss of large multimers and an increase of small multimers in an HUS plasma sample compared with the control and colitis plasma samples. In the gel analysis, smaller multimers moved faster toward the bottom of the gel. For reference, the location of IgM is indicated on the left. The colitis sample, obtained from the same patient, contained the entire size spectrum of multimers. On densitometric analysis, the fraction of large multimers (those larger than the four smallest bands) was determined to be slightly decreased to $89 \%$. B, levels of the large multimer fraction in seven normal children, 37 colitis, 16 HUS, and 14 normal adult plasma samples. The large multimer fraction decreased to $<91 \%$, or $3 \mathrm{SD}$ below the mean, of the controls, in all 16 HUS and in six colitis samples. In each of the eight patients $(\triangle)$ who were studied at both stages, the fraction of large multimers decreased at the stage of HUS. 
During the colitis stage, the multimer distribution did not differ visually from that of control plasma, but, by densitometry, the large multimer fraction was decreased to $89 \%$. When HUS ensued, the large multimer fraction in this patient further decreased to $62 \%$.

The fractions of the large multimers in each of the samples studied are depicted in Figure $2 B$. Of the 37 patients in the colitis stage of infection, the large multimer fraction was $96 \pm$ $8 \%$, compared with $100 \pm 3 \%$ in the seven control children and $101 \pm 3 \%$ in 14 normal adults. No difference in the large multimer fraction was detected between the 29 patients who had uncomplicated infection (96 $\pm 8 \%$ ) and the eight patients who subsequently developed HUS ( $98 \pm 6 \%, p>0.45)$. In six of the 37 samples obtained at the colitis stage (including one from a patient who subsequently developed HUS), the large multimer fraction was $<91 \%$, i.e. $>3$ SDs below the mean of the controls. Of the 16 patients investigated at the onset of HUS, the large multimer fraction was $79 \pm 10 \%$. This value was significantly decreased, compared with the control and with the colitis samples $(p<0.001)$. In the eight patients who were studied in both colitis and HUS stages, the onset of HUS was associated with a decrease in the large multimer fraction from $98 \pm 6 \%$ to $77 \pm 12 \%(p<0.001)$. To determine whether the decrease of von Willebrand factor multimer size was related to the thrombotic process, the values of the large multimer fraction were plotted against the corresponding platelet counts (Fig. 3). The graph demonstrates a correlation between a decrease in platelet counts and a decrease in the fractions of large multimers $(p<0.001)$. However, as the graph demonstrates, the relation between these two variables was complex, and in a linear regression analysis, the coefficient of correlation was 0.56 .

Among the patients investigated at the colitis stage of infection, the fraction of large von Willebrand factor multimer was $96 \pm 8 \%$ in the 24 children whose infecting isolates contained stx 1 and $s t x 2$, and was $98 \pm 7 \%$ in the 13 children whose isolates contained $s t x 2$ but not stxl $(p>0.4)$. Among the patients investigated at the beginning of HUS, the large von

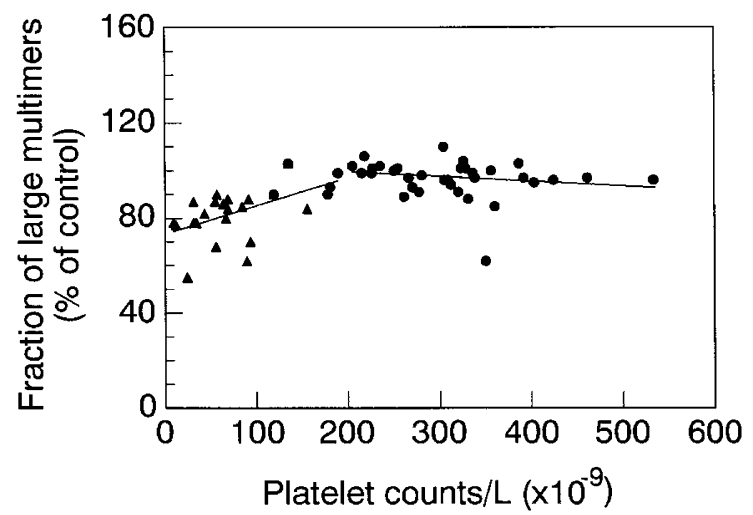

Fig. 3. A decrease in the platelet counts was associated with a decrease in the large multimers. The fractions of large multimers are plotted against the platelet counts for patients with colitis $(\bullet)$ and patients with HUS ( $\bullet$ ). For the entire group, a decrease in the platelet count correlated with a decrease in the fraction of the large multimers $(p<0.001)$. The lines depict the regression for samples with platelet counts above or below $200 \times 10^{9} / \mathrm{L}$. In some patients in both groups, a dramatic decrement in the large multimer fraction was noted.
Willebrand factor multimer fraction was $80 \pm 12 \%$ in the 10 children whose infecting isolates contained stx 1 and stx 2 and was $78 \pm 8 \%$ in the six children whose isolates contained stx2 but not stxl $(p>0.5)$.

Von Willebrand factor proteolysis. To determine whether enhanced cleavage contributes to the decrease of large von Willebrand factor multimers, we analyzed the concentration of proteolytic fragments in the plasma samples by SDS PAGE and immunoblotting.

Plasma from one representative patient is demonstrated in Figure $4 A$. Compared with samples obtained during the colitis stage of infection (lane 2), the $350-\mathrm{kD}$ and $200-\mathrm{kD}$ species are clearly increased at the HUS stage (lane 3).

Thirty-six samples obtained at the colitis stage and 16 samples obtained at the HUS stage were available for proteolysis analysis (plasma from one colitis patient had insufficient volume for proteolysis analysis). The results of the $350-\mathrm{kD}$ band density are depicted in Figure $4 B$. The $350-\mathrm{kD}$ band density increased from $147 \pm 65 \%$ of control among the colitis samples to $495 \pm 193 \%$ among the HUS samples $(p<0.001)$. Similarly, the $200-\mathrm{kD}$ band density increased from $133 \pm 68 \%$ of control among the colitis samples to $471 \pm 225 \%$ among the HUS samples $(p<0.001$; individual data not shown). In the eight patients who were investigated at both stages, the $350-\mathrm{kD}$ band density increased from $176 \pm 65 \%$ at colitis to $492 \pm$ $189 \%$ at HUS $(p<0.001)$. In these eight patients, the $200-\mathrm{kD}$ band also increased from $174 \pm 63 \%$ at colitis to $374 \pm 110 \%$ at HUS $(p<0.001$; individual data not shown).

To determine whether the observed increase in the fragments was solely attributable to an increase in von Willebrand factor antigen concentrations, the von Willebrand factor antigen levels in the two groups of plasma samples were determined by enzyme immunoassay (Fig. 5A). The von Willebrand factor antigen concentration increased from $137 \pm 37 \%$ of the control value in the 37 samples obtained at the colitis stage to $230 \pm$ $74 \%(p<0.001)$ in the 16 samples obtained at the HUS stage.
A

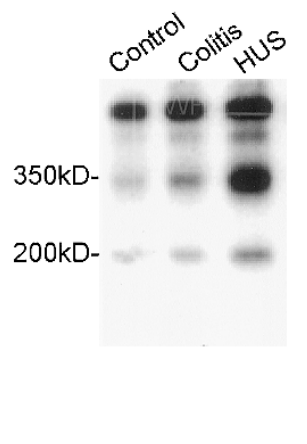

B

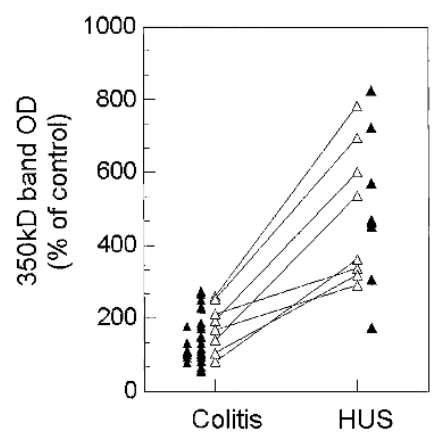

Fig. 4. Increased von Willebrand factor proteolysis in association with HUS caused by E. coli O157:H7 infection. A, immunoblot of SDS PAGE depicting the $350-\mathrm{kD}$ and $200-\mathrm{kD}$ von Willebrand factor fragments in a control plasma sample and in the colitis and HUS plasma samples of a patient studied at both stages. $B$, distribution of the OD of the $350-\mathrm{kD}$ band in 37 colitis and 16 HUS plasma samples. As a group, the $350-\mathrm{kD}$ band OD was higher in the HUS samples than in colitis samples $(p<0.001)$. In each of the eight individuals studied at both stages $(\triangle)$, the onset of HUS was associated with an increased $350-\mathrm{kD}$ band OD $(p<0.001)$. Analysis of the $200-\mathrm{kD}$ band produced similar results (data not shown). 
In the eight patients investigated at both stages, the von Willebrand factor antigen level increased from $184 \pm 65 \%$ to $234 \pm 62 \%(p=0.0424)$. However, an increase was not observed in two patients and was $<30 \%$ in two additional patients. In each of these eight patients, the ratio of the $350-\mathrm{kD}$ band density at the HUS stage to the density of the same band at the colitis stage $(313 \pm 84 \%)$ was higher than the ratio of von Willebrand factor antigen levels $(148 \pm 62 \%, p<0.002$; Fig. 5B).

The ratios of the $350-\mathrm{kD}$ and $200-\mathrm{kD}$ bands to von Willebrand factor antigen $(222 \pm 73 \%$ and $217 \pm 129 \%$, respectively) were higher for the 16 HUS samples than for the 36 colitis samples (109 $\pm 44 \%$ and $101 \pm 58 \%, p<0.001$ and $p$ $<0.004$, respectively). These data show that von Willebrand factor fragments in the HUS samples remained elevated after adjustment for von Willebrand factor concentrations.

To demonstrate further that cleavage contributed to the decrease of large von Willebrand factor multimers, we plotted the $350-\mathrm{kD}$ band density against the fraction of large multimers for all the samples (Fig. 6A). The coefficient of correlation between these two variables was $-0.552(p<0.001)$. The coefficient of correlation between the 200-kD band density and the fraction of large multimers for all the samples was -0.628 $(p<0.001$; individual data not shown). To account for the increase of the von Willebrand factor antigen level in association with the onset of HUS, the $350-\mathrm{kD}$ band density was divided by the von Willebrand factor antigen concentration. As shown in Figure $6 B$, the correlation between the $350-\mathrm{kD}$ band density/von Willebrand factor antigen ratio and the fraction of large multimers remained highly significant (coefficient of correlation, $-0.466 ; p<0.001)$. A similar correlation $(-0.506 ; p<0.001)$ was also observed between $200-\mathrm{kD}$ band density/von Willebrand factor antigen ratio and the fraction of large multimers (individual data not shown).

Taken together, these data indicate that the increase in the concentration of proteolytic fragments is out of proportion to
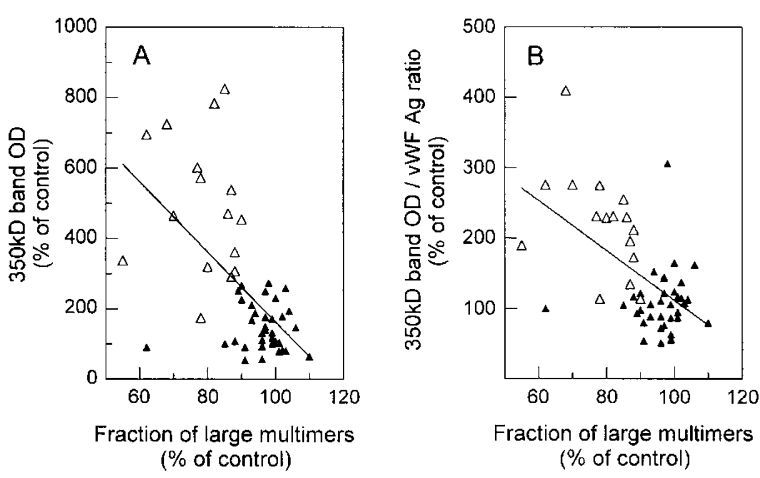

Fig. 6. A decrease in the large von Willebrand factor multimers was associated with an increase in von Willebrand factor proteolytic fragments. $A$, plot of the $350-\mathrm{kD}$ band density against the fraction of large multimers for 36 colitis samples $(\Delta)$ and 16 HUS $(\triangle)$ samples. The regression line indicates that a decrease of the large multimers correlates with an increase in the $350-\mathrm{kD}$ band density $(p<0.001)$. $B, 350-\mathrm{kD}$ band $\mathrm{OD} /$ von Willebrand factor $(\nu W F)$ antigen $(\mathrm{Ag})$ ratio also inversely correlated with the fraction of large multimers $(p<$ 0.001 ). Analysis of the $200-\mathrm{kD}$ band density produced similar results (data not shown).

the increase in the plasma concentration of von Willebrand factor antigen, which can be elevated as an acute phase reactant.

Immunochemical findings of the thrombotic lesions. To determine whether fibrin and von Willebrand factor were present in the thrombotic lesions of HUS, we examined postmortem renal sections from four children who died of HUS after hemorrhagic colitis caused by Shiga toxin-producing $E$. coli. As controls, renal tissue from a patient with disseminating intravascular coagulation and brain tissue from a patient with TTP were also studied. As illustrated in Figure 7, thrombi in the patient with disseminating intravascular coagulation and the patient with HUS contained fibrin (pink) but not von Willebrand factor. In contrast, the lesions of TTP stained
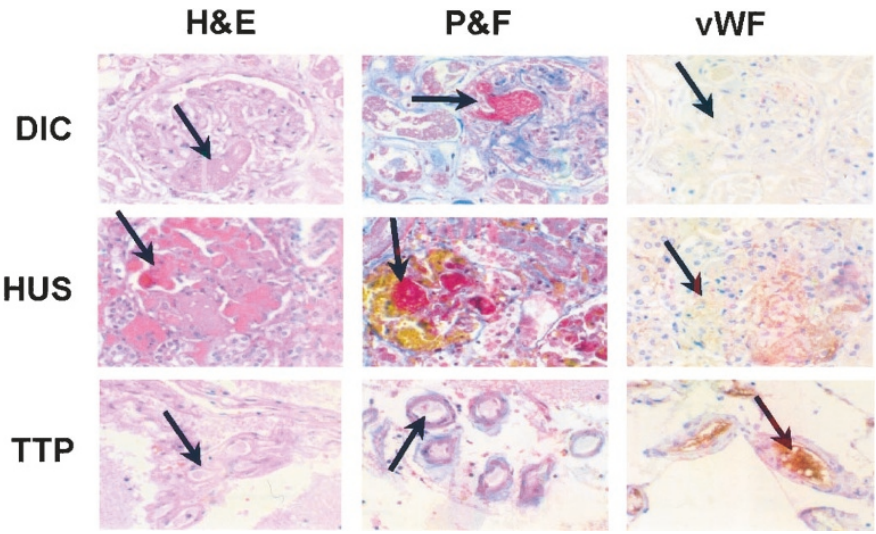

Fig. 7. Analysis of the glomerular microthrombi (arrows) in postmortem sections from a child who died from E. coli O157:H7-associated HUS. The thrombi contain fibrin (pink) but not von Willebrand factor $(v W F)$. The glomerular endothelial cells were weakly positive for von Willebrand factor (brown). In comparison, postmortem renal sections from a patient with disseminating intravascular coagulation $(D I C)$ demonstrate microthrombi with fibrin, and postmortem brain sections from a patient with TTP contained platelets (blue-gray) and von Willebrand factor. $H \& E$, hematoxylin and eosin stain; $P \& F$, platelet and fibrin stain of Carstairs (23). 
positive for platelets (blue-gray) but negative for fibrin. In TTP, von Willebrand factor was identified within the microthrombus (brown).

The postmortem kidney sections of three of the four patients with HUS demonstrated abundant glomerular microthrombi that stained positive for fibrin. In these patients, von Willebrand factor was present within endothelial cells (a normal finding) but not in the thrombi. In one patient, thrombi were detected in the arterioles and small arteries but not in the glomeruli. The thrombi in this patient stained positive for fibrin; some of the lesions also contained von Willebrand factor.

\section{DISCUSSION}

We have demonstrated that in well-characterized HUS caused by E. coli $\mathrm{O} 157: \mathrm{H} 7$, there is no deficiency of circulating von Willebrand factor-cleaving metalloprotease activity. We further demonstrate that fibrin, and not von Willebrand factor, is abundant in glomerular thrombi of E. coli O157:H7associated HUS. In each of the HUS cases, the size of von Willebrand factor, as represented by the large multimer fraction, was decreased, and in none of these cases were ultralarge multimers detected. The change in von Willebrand factor multimers is specific for HUS because patients with renal failure from other causes have normal circulating von Willebrand factor multimers (26).

Conflicting data exist in the literature regarding von Willebrand factor size in childhood HUS. Moake et al. (14) reported a relative decrease in large multimers in each of seven patients with HUS, but subsequently Moake (15) reported that 24 of 51 patients with HUS had a relative decrease in large multimers, and three had unusually large multimers. Rose et al. (16) reported that an increase of large multimers was observed in each of the 13 patients with diarrhea-associated HUS. Mannucci et al. (17) noted the presence of unusually large multimers in four patients with HUS, but the proteolytic fragments were also increased.

Disorders termed as HUS comprise a heterogeneous group with different pathogenetic mechanisms (2-4). In previous studies of von Willebrand factor abnormalities in HUS, the cause of the HUS and the stage of illness that the patients were in were not always provided, and the criteria for distinguishing between HUS and TTP were incompletely defined. Therefore, different subsets of HUS might have been included, including some patients who might have had TTP. Because of these reasons, it is difficult to compare our findings with those in the literature. Furthermore, because the size distribution of von Willebrand factor multimers is not analyzed in a quantitative manner, subtle changes might have been overlooked. In fact, our interpretation of the multimer patterns published by Rose $e t$ al. (16) suggests that small multimers are increased. In future studies, it will be instructive to include results of microbiologic studies of the stools and a determination of the von Willebrand factor-cleaving metalloprotease activity as part of the case definition and characterization of thrombotic microangiopathies.
The correlation between the fractions of large multimers and the platelet counts in our patients suggests that the decrease of large von Willebrand factor multimers and the process of thrombocytopenia are related. Large von Willebrand factor multimers may participate in, and be consumed during, the evolution of thrombosis as HUS develops. Alternatively or additionally, large von Willebrand factor molecules may be unfolded (11) by abnormal shear stress in the microvascular circulation and become more susceptible to proteolysis (8). The latter scenario is supported by the increase of the von Willebrand factor proteolytic fragments that we have detected in infected patients at the onset of HUS. In most cases, the magnitude of the increase of proteolytic fragments, as represented by the $350-\mathrm{kD}$ and $200-\mathrm{kD}$ species, is higher than that expected from the increase in von Willebrand factor antigen concentration and is directly correlated with a decrease in the fraction of large multimers.

The reason for the presence of von Willebrand factor in the arterial and arteriolar, but not in the glomerular, thrombi, remains speculative. Thrombotic lesions in different parts of the vasculature probably increase the shear rate of von Willebrand factor differently. The resulting von Willebrand factor proteolysis will be variable. Based on the heterogeneous thrombus components, we further speculate that although von Willebrand factor metalloprotease is not deficient in HUS, in some cases von Willebrand factor may be involved in the thrombotic process, leading to a consumption of large von Willebrand factor multimer. This process may explain why the decrease of large multimers is sometimes more than what would have been expected from the severity of thrombocytopenia (Fig. 3) and, in occasional cases, the decrease is not associated with a clear increase in von Willebrand factor proteolytic fragments (Fig. 6).

Our immunohistochemical findings are consistent with those of Asada et al. (27), who detected abundant von Willebrand factor but little or no fibrin in the thrombi of TTP, and those of Habib (1), who detected prominent fibrin in the thrombi of HUS.

Our data also shed light on the vascular response to $E$. coli O157:H7 infection, even in the absence of HUS. For example, we detect a decrease in the size of von Willebrand factor multimers in a subset of patients with hemorrhagic colitis whose illness resolves without progression to overt HUS. These data suggest that the colitis may, at least in some patients, be accompanied by microvascular thrombosis, and that this process resolves without evolving into HUS. Indeed, capillary platelet-fibrin thrombi have been detected in the colonic mucosa of classic HUS (28-31) and in chimpanzees infused with Shiga toxins (32).

Finally, our data have practical implications. In TTP, the deficiency of the circulating von Willebrand factor metalloprotease now provides a rational basis for the use of plasma exchange or plasma infusion to treat this disorder. However, the normal von Willebrand factor-cleaving metalloprotease activity in children with $E$. coli $\mathrm{O} 157: \mathrm{H} 7$-associated HUS lends no support to the use of these modalities in the treatment of this disorder. In fact, two clinical trials of plasma therapy in pediatric cases of HUS fail to detect definitive benefits of 
plasma therapy on survival, course of hematologic changes, or long-term recovery of renal functions $(33,34)$.

In conclusion, our data do not support an etiologic role for von Willebrand factor metalloprotease deficiency in the formation of renal microthrombi in HUS and provide evidence in support of a distinction in pathophysiology between $E$. coli O157:H7-associated HUS and TTP (2-4). Furthermore, E. coli O157:H7-associated HUS is accompanied by increased proteolysis of von Willebrand factor. A decrease in von Willebrand factor large multimers should diminish the platelet-aggregating activity of this molecule, so enhanced proteolysis of circulating von Willebrand factor multimers may explain in part the absence of von Willebrand factor in the glomerular thrombi of HUS.

Acknowledgments. The authors thank participating laboratories, nurses, physicians, and families of infected children and of controls for their cooperation with our studies. We also thank Dr. Kathleen Patterson for access to tissues, Anping Li for technical assistance, and Kaye Green for assistance in manuscript preparation.

\section{REFERENCES}

1. Habib R 1992 Pathology of the hemolytic uremic syndrome. In: Kaplan BS, Trompeter RS, Moake JL (eds) Hemolytic Uremic Syndrome and Thrombotic Thrombocytopenic Purpura. Marcel Dekker, New York, pp 315-353

2. Kaplan BS, Drummond KN 1978 The hemolytic-uremic syndrome is a syndrome N Engl J Med 298:964-966

3. Kaplan BS, Meyers KE, Schulman SL 1998 The pathogenesis and treatment of hemolytic uremic syndrome. J Am Soc Nephrol 9:1126-1133

4. Taylor CM, Howie AJ, Williams JM 1999 No common final pathogenetic pathway in haemolytic uraemic syndromes. Nephrol Dial Transplant 14:1100-1102

5. Tarr PI, Neill MA, Clausen CR, Watkins SL, Christie DL, Hickman RO 1990 Escherichia coli $\mathrm{O} 157: \mathrm{H} 7$ and the hemolytic uremic syndrome: importance of early cultures in establishing the etiology. J Infect Dis 162:553-556

6. Koster F, Levin J, Walker L, Tung KS, Gilman RH, Rahaman MM, Majid MA, Islam S, Williams Jr RC 1978 Hemolytic-uremic syndrome after shigellosis. N Engl J Med 298:927-933

7. Dent JA, Berkowitz SD, Ware J, Kasper CK, Rugggeri ZM 1990 Identification of a cleavage site directing the immunochemical detection of molecular abnormalities in type IIA von Willebrand factor. Proc Natl Acad Sci USA 87:6306-6310

8. Tsai HM, Sussman II, Nagel RL 1994 Shear stress enhances the proteolysis of von Willebrand factor in normal plasma. Blood 83:2171-2179

9. Furlan M, Robles R, Lämlie B 1996 Partial purification and characterization of a protease from human plasma cleaving von Willebrand factor to fragments produced by in vivo proteolysis. Blood 87:4223-4234

10. Tsai HM 1996 Physiologic cleavage of von Willebrand factor by a plasma protease is dependent on its conformation and requires calcium ion. Blood 87:4235-4244

11. Siedlecki CA, Lestini BJ, Kottke-Marchant KK, Eppell SJ, Wilson DL, Marchant RE 1996 Shear-dependent changes in the three dimensional structure of human von Willebrand factor. Blood 88:2939-2950
12. Tsai HM, Lian EC-Y 1998 Antibodies to von Willebrand factor-cleaving protease in acute thrombotic thrombocytopenic purpura. N Engl J Med 339:1585-1594

13. Furlan M, Robles R, Galbusera M, Remuzzi G, Kyrle PA, Brenner B, Krause M, Scharrer I, Aumann V, Mittler U, Solenthaler M, Lämmle B 1998 von Willebrand factor-cleaving protease in thrombotic thrombocytopenic purpura and the hemolytic uremic syndrome. N Engl J Med 339:1578-1584

14. Moake JL, Byrnes JJ, Troll J, Rudy CK, Weinstein MJ, Colannino NM, Hong SL 1984 Abnormal VIII: von Willebrand factor patterns in the plasma of patients with the hemolytic-uremic syndrome. Blood 64:592-598

15. Moake JL 1992 von Willebrand factor abnormalities in thrombotic thrombocytopenic purpura and the hemolytic uremic syndrome. In: Kaplan BS, Trompeter RS, Moake JL (eds) Hemolytic Uremic Syndrome and Thrombotic Thrombocytopenic Purpura. Marcel Dekker, New York, pp 459-471

16. Rose PE, Enayat SM, Sunderland RR, Short PE, Williams CE, Hill FG 1984 Abnormalities of factor VIII related protein multimers in the haemolytic uraemic syndrome. Arch Dis Child 59:1135-1140

17. Mannucci PM, Lombardi R, Lattuada A, Ruggenenti P, Vigano GLA, Barbui T, Remuzzi G 1989 Enhanced proteolysis of plasma von Willebrand factor in thrombotic thrombocytopenic purpura and the hemolytic uremic syndrome. Blood 74:978-983

18. Wong CS, Jelacic S, Habeeb RL, Watkins SL, Tarr PI 2000 The risk of hemolytic uremic syndrome after antibiotic treatment of Escherichia coli $\mathrm{O} 157: \mathrm{H} 7$ infections. N Engl J Med 342:1930-1936

19. Sambrook J, Fritsch EF, Maniatis T 1989 Molecular cloning: a laboratory manual, 2nd Ed. Cold Spring Harbor Laboratory Press, Cold Spring Harbor, NY

20. Newland JW, Neill RJ 1988 DNA probes for Shiga-like toxins I and II and for toxin-converting bacteriophages. J Clin Microbiol 26:1292-1297

21. Tarr PI, Fouser LS, Stapleton AE, Wilson RA, Kim HH, Vary Jr JJ, Clausen CR 1996 Hemolytic-uremic syndrome in a six-year-old girl after a urinary tract infection with Shiga-toxin-producing Escherichia coli O103:H2. N Engl J Med 335:635-638

22. Tarr PI, Neill MA, Clausen CR, Newland JW, Neill RJ, Moseley SL 1989 Genotypic variation in pathogenic Escherichia coli $\mathrm{O} 157: \mathrm{H} 7$ isolated from patients in Washington, 1984-1987. J Infect Dis 159:344-347

23. Carstairs KC 1965 The identification of platelets and platelet antigens in histological sections. J Pathol Bacteriol 90:225-231

24. Glantz SA 1997 Primer of Biostatistics, 4th Ed. McGraw-Hill Health Professions, New York, pp191-244

25. Bennett C, Connors J, Carwile J, Moake J, Bell W, Tarantolo S, McCarthy L, Sarode R, Hatfield A, Michalets E, Feldman M, Davidson C, Tsai H 2000 Thrombotic thrombocytopenic purpura associated with clopidogrel. N Engl J Med 342:1773-1777

26. Castaman G, Rodeghiero F, Lattuada A, La-Greca G, Mannucci PM 1993 Multimeric pattern of plasma and platelet von Willebrand factor is normal in uremic patients. Am J Hematol 44:266-269

27. Asada Y, Sumiyoshi A, Hayashi T, Suzumiya J, Kaketani K 1985 Immunohistochemistry of vascular lesions in thrombotic thrombocytopenic purpura with special reference to factor VIII antigen. Thromb Res 38:469-479

28. Gianantiono CA, Vitacco M, Mendilaharzu F, Gallo GE, Sojo ET 1973 The hemolytic uremic syndrome. Nephron 11:174-192

29. Tochen ML, Campbell JR 1977 Colitis in children with the hemolytic uremic syndrome. J Pediatr Surg 12:213-217

30. Whitington PF, Freidman AL, Chesney RW 1979 Gastrointestinal disease in the hemolytic-uremic syndrome. Gastroenterology 76:728-733

31. Morrison DM, Tyrrell LJ, Jewell LD 1986 Colonic biopsy in verotoxin-induced hemorrhagic colitis and thrombotic thrombocytopenic purpura. Am J Clin Pathol $86: 108-112$

32. Taylor Jr FJ, Tesh VL, DeBault L, Li A, Chang AC, Kosanke SD, Pysher TJ, Siegler RL 1999 Characterization of the baboon responses to Shiga-like toxin: descriptive study of a new primate model of toxic responses to Stx-1. Am J Pathol 154:12851299

33. Loirat C, Sonsino E, Hinglais N, Jais JP, Landais P, Fermanian J 1988 Treatment of the childhood haemolytic uraemic syndrome with plasma. Pediatr Nephrol 2:279-285 34. Rizzoni G, Claris Appiani A, Edefonti A, Facchin P, Franchini F, Gusmano R, Imbasciati E, Pavanello L, Perfumo F, Remuzzi G 1988 Plasma infusion for hemolytic-uremic syndrome in children: results of a multicenter controlled trial. J Pediatr 112:284-290 